\title{
Prevalence of Overlap Syndrome. Obstructive Sleep Apnea in Patients with Chronic Obstructive Pulmonary Disease. A study protocol.
}

\author{
Julio Cesar Mendes Oliveira,2, Anderson Soares Silva', Ana Carolina Negrinho de Oliveira Beloto², Jéssica Julioti Urbano', Ezequiel \\ Fernandes Oliveira', Eduardo Araujo Perez³ ${ }^{3}$ Roger Andre Oliveira Peixoto', Israel Reis Santos', Luis Vicente Franco Oliveira'.
}

\begin{abstract}
Introduction: Chronic obstructive pulmonary disease (COPD) and Obstructive sleep apnea (OSA) cause an inflammatory response and hypoxia. Patients who have both conditions have increased morbidity and mortality. Overlap syndrome between OSA and COPD is important but under-recognised. Objectives: We aimed to determine the prevalence and severity of overlap syndrome in patients with COPD through the overnight standard polysomnography. Methods/Design: A consecutive single-center cross-sectional study will be performed. The design, conduct and report of this study followed the guidelines of the Strengthening the Reporting of Observational Studies in Epidemiology (STROBE) statement. The sample will be of convenience, recruited consecutively with respiratory complaints that seek care in a private clinic specializing in pulmonology in the city of Cascavel, in the state of Paraná, from September 2016 to July 2017. All subjects will be submitted to the same evaluation protocol described subsequently. Initially will be collected data on baseline demographic, anthropometric and clinical aspects, including body mass index, neck, hip and waist circumferences, respiratory and cardiac rates, peripheral blood pressure, and BODE index. After this phase of the study, patients will perform lung function tests through petismography, sleep studies using the standard overnight PSG, and respond to the Berlin and Pittsburgh questionnaires, and Epworth Sleepiness Scale.
\end{abstract}

Keywords: COPD, respiratory sleep disorders, apnea obstructive sleep, Overlap syndrome, Pulmonary Rehabilitation, Epidemiology.

Trial Registration: This protocol study has been approved by the Research Ethics Committee of the Nove de Julho University (Brazil), process $n^{\circ} 370474 / 2010$, and will be registered on ClinicalTrials.gov.

\section{BACKGROUND}

Chronic Obstructive Pulmonary Disease (COPD) is a common, preventable and treatable disease that is characterized by persistent respiratory symptoms and airflow limitation that is due to airway and/or alveolar abnormalities usually caused by significant exposure to noxious particles or gases. . $^{(1,2,3)}$

COPD is currently the fourth leading cause of death in the world but is projected to be the 3rd leading cause of death by $2020 .{ }^{(4)}$ More than 3 million people died of COPD in 2012 counting $6 \%$ of all deaths globally. COPD represents an important public health challenge that is preventable and treatable. COPD is a major cause of chronic morbidity and mortality throughout the world; many people suffer

from this disease for years, and die prematurely from it or its complications. Globally, the COPD burden is projected to increase in incoming decades because of continued exposure to COPD risk factors and aging of the population. ${ }^{(5)}$

Overlap syndrome is a term used to describe the coexistence of obstructive sleep apnea (OSA) and COPD, and was first time proposed by the researcher David Flenley, when he estimated that this association of two common diseases probably involved many patients. ${ }^{(6)}$ Indeed, research has indicated a high prevalence of OSA in COPD patients, including one recent study which shows up that $66 \%$ of the COPD patients, who enrolled in pulmonary rehabilitation, tested positive for OSA. ${ }^{(7)}$

\footnotetext{
*Corresponding author: Luis Vicente Franco de Oliveira. Sleep Laboratory, Universidade Nove de Julho (UNINOVE), São Paulo, (SP), Brasil. oliveira.Ivf@uninove.br

1Universidade Nove de Julho (UNINOVE), São Paulo, (SP), Brasil.

Full list of author information is available at the end of the article.

FINANCIAL SUPPORT: The authors would like to acknowledge the technical infrastructure support provided by Lung Institute of Cascavel (Brazil) and by Sleep Laboratory of Nove de Julho University - UNINOVE (Brazil). The Sleep Laboratory receives funding from the Nove de Julho University (Brazil) and research projects approved by the Brazilian fostering agencies Fundaçao de Amparo a Pesquisa do Estado de Sao Paulo (local acronym FAPESP; protocol no. 2003/01810-4). JCMO receives grants of Coordenação de Apoio ao Pessoal de Nível Superior (CAPES/PROSUP); LVFO receive grants Research Productivity, modality PQ1B; process no. 313053/20146 of Conselho Nacional de Desenvolvimento Cientifico e Tecnologico (local acronym CNPq), Brazil.
}

Received: December 01, 2016; Accepted: December 09, 2016 
Furthermore, patients with overlap syndrome may experience worsening symptoms of COPD. ${ }^{(8)}$

COPD has an estimated prevalence in U.S. adults of $13.9 \%^{9,10}$ and OSA, a sleep disorder hallmarked by repeated episodes of upper airway closure, affects $9 \%$ to $26 \%$ of the U.S. adult population. ${ }^{(11)}$ The term "overlap syndrome" has been used to describe the association of both conditions in a single patient. ${ }^{(6)}$

Patients with overlap syndrome have a worse prognosis compared with COPD or OSA. During sleep, patients with both COPD and OSA suffer more frequent episodes of oxygen desaturation and have more sleep time with hypoxemia and hypercapnia than OSA patients without COPD. ${ }^{(12)}$ The apneic events in patients with combined OSA and COPD have more profound hypoxemia and more cardiac arrhythmias. (13) Additionally, patients with combined COPD and OSA are more likely to develop daytime pulmonary hypertension than patients with just OSA or COPD alone. ${ }^{(14,15)}$

Among the main clinical consequences of the overlap syndrome, we give attention to the increased incidence of exacerbations, prolonged oxygen desaturation during sleep, which can lead to increased systemic and pulmonary blood pressure. This facts increases the risk of pulmonary hypertension, right heart failure and/or cor pulmonale. ${ }^{(16,17)}$

Hawrylkiewicz et al. (2004), showed that the prevalence of pulmonary hypertension in OSA patients was $16 \%$ compared to $86 \%$ in overlap syndrome patients. ${ }^{(18)}$ Other studies, showed higher mortality rates compared to those with either COPD or OSA alone. ${ }^{(19-21)}$ A study conducted by Lavie et al. (1995), founded that mortality rates were seven times higher in the overlap syndrome population, (22) while McNicholas and Fitzgerald (1984) observed the higher incidence of mortality at night study ${ }^{(23)}$, and other study reported a poorer quality of life. ${ }^{(24)}$

Independently, COPD and OSA are highly prevalent disorders $^{(25-27)}$, so the risk of overlap syndrome should be relatively high. Recent studies estimate that the prevalence of COPD ${ }^{(25,28)}$ is approximately $10 \%$ while OSA compromises about $9 \%$ to $26 \%$ of the adult population. ${ }^{(27,29,30)}$ The overlap syndrome presents considerable prevalence among patients with COPD $^{(7,31-35)}$ and carries additional prognostic implications relating to worsening respiratory failure, cardiovascular and other co-morbidities, and ultimately survival. ${ }^{(36)}$

OSA and COPD are common inflammatory systemic disorders with increasing prevalence. Not well documented in the literature, the overlap syndrome leads to increased disease severity, morbidity and mortality. Identifying and appropriately treating OSA in patients with COPD can improve disease outcomes, it is very important to clinicians recognize in advance patients who are presenting signs and symptoms of sleep-disordered breathing, especially those with COPD, so they may be treated appropriately.

\section{AIMS AND HYPOTHESIS}

\section{STUDY OBJECTIVES}

Main objective

To determine the prevalence and severity of Overlap syndrome in patients with COPD, through the overnight standard polysomnography.

\section{Secondary objectives}

- To verify the prevalence of excessive daytime sleepiness, the risk for OSA, and quality of sleep through the Epworth Sleepiness Scale, Berlin questionnaire, and Pittsburgh Questionnaire in patients with COPD;

- Identify whether the presence of OSA in patients with COPD is correlated with age, neck and waist circumference, pulmonary function, and BODE index.

Our hypothesis is that there should be a correlation between the severity of OSA and the physiological and clinical variables from the patients with COPD.

\section{STUDY DESIGN AND SETTING}

The design, conduct and report of this study followed the guidelines of the Strengthening the Reporting of Observational Studies in Epidemiology (STROBE) ${ }^{(37)}$ statement, according to figure 1. A consecutive, single-center, cross-sectional study will be performed, to investigate the prevalence and severity of Overlap syndrome in patients with COPD. The sample will be of convenience, composed of patients seeking care in a private clinic specialized in pulmonology in the city of Cascavel, extreme west of the state of Paraná.

\section{ETHICAL AND LEGAL ASPECTS}

This protocol study has been approved by the Research Ethics Committee of the Nove de Julho University (Brazil), process $n^{\circ} 370474 / 2010$. The study will be conducted in accordance with the ethical standards established in 1961 by Declaration of Helsinki and will be in accordance with the Regulatory Guidelines and Standards of Research Involving Human Subjects of Comissão Nacional de Etica em Pesquisa (CONEP) do Ministerio da Saude published in December, 2012. The signing of the free and informed consent will be required from all patients, and they will be allowed to withdraw from the study at any time without any negative consequences. Patients with severe clinical problems will be referred to the appropriate treatment aimed at their health status and quality of life.

\section{SUBJECTS AND RECRUITMENT PROCEDURE}

Subjects will be recruited consecutively with respiratory complaints that seek care in a private clinic specializing in pulmonology in the city of Cascavel, in the state of Paraná, from 


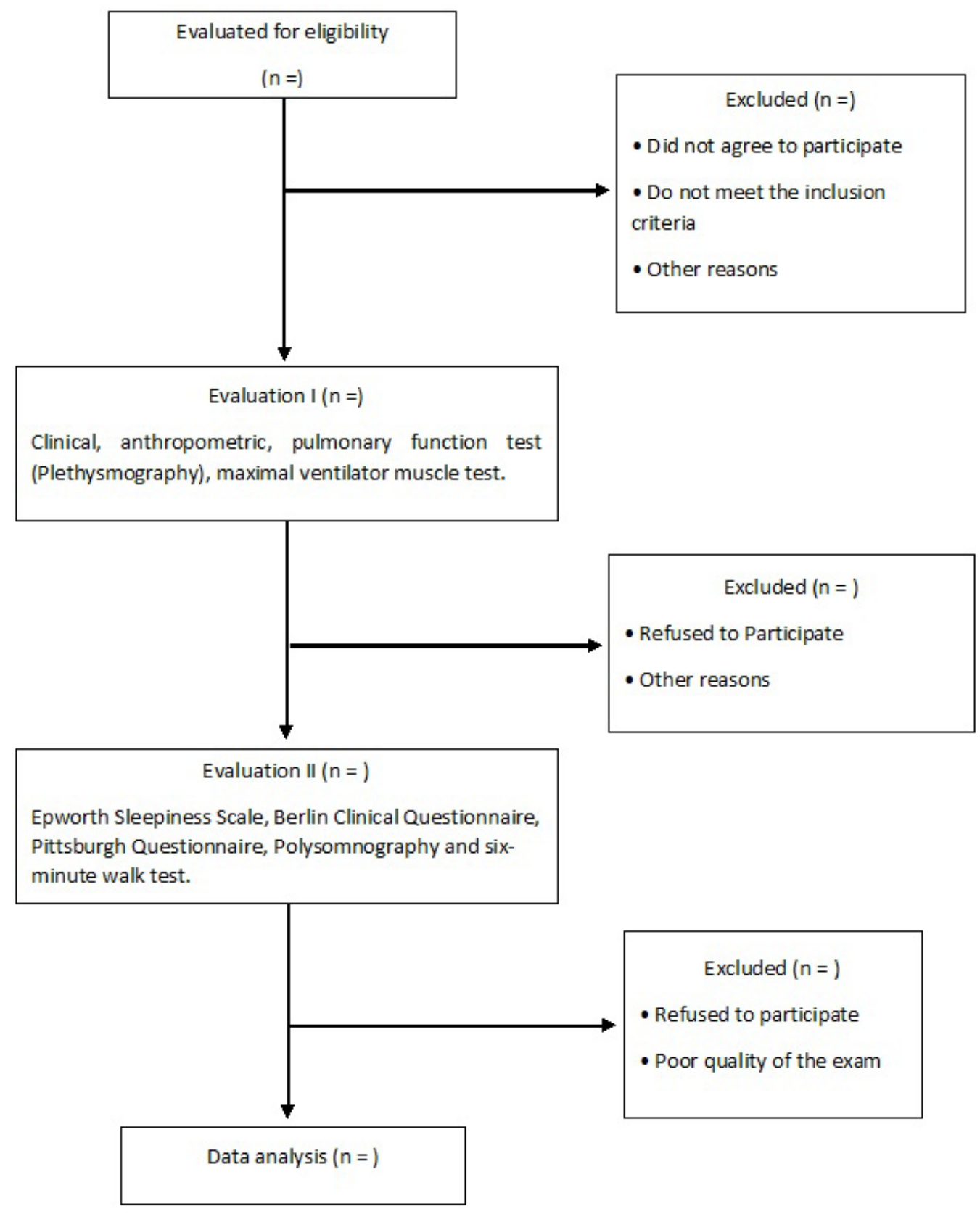

Figure 1. Study flowchart

September 2016 to July 2017. Adult patients diagnosed with COPD based on the Global Initiative for Chronic Obstructive Lung Disease (GOLD) ${ }^{(38,39)}$ definition and spirometric criteria, clinically stable for at least 2 months will be invited to participate in the study. None of the participants may have previously participated in a pulmonary rehabilitation program.

\section{Eligibility criteria}

The COPD patients will be enrolled in this prospective study according to the following inclusion criteria. Clinical diagnosis of COPD based on the GOLD guideline. ${ }^{(39)}$ Patients will be grouped according to FEV1\% values to assess the severity of airflow obstruction. Patients with FEV1>80\% were defined as having mild, FEV1 50\%-79\% moderate, FEV1 30\%-49\% severe, while $\mathrm{FEV} 1<30 \%$ as very severe airflow obstruction, and have not had an episode of exacerbation for at least 4 weeks before being involved in the study. The clinical stability criterion was defined as being of stable symptomatology (dyspnea, volume or color of secretion), with no therapeutic variation, no use of antibiotics and steroids, unless it was of chronic use.

According to the exclusion criteria, patients with lung diseases other than COPD (e.g., asthma or bronchiectasis), Cor pulmonale, respiratory analeptic utilization, uncontrolled 
hypertension, unstable angina, left heart failure, congenital heart disease, severe comorbidity such as hepatic cirrhosis, chronic renal failure, uncontrolled diabetes and active malignancy, neuromuscular disorders, sequelae of stroke and subjects previously submitted to pneumonectomies or other chest surgeries were excluded. The presence of comorbidities will not be considered as exclusion criteria, since they are stable and the great majority of COPDs are elderly, commonly affected by multiple comorbidities.

\section{CLINICAL EVALUATION}

All subjects will be submitted to the same evaluation protocol described subsequently. Initially, will be collected data on demographic, anthropometric and clinical baseline for all recruited patients, including body weight $(\mathrm{kg})$, height (m), BMI, neck, hip and waist circumferences $(\mathrm{cm})$, respiratory and cardiac rates, and peripheral blood pressure. Arterial blood samples will be obtained in the supine position by radial puncture. After this phase of the study, patients will perform lung function tests through petismography, sleep studies using the standard overnight PSG, and respond to the Berlin and Pittsburgh questionnaires, and Epworth Sleepiness Scale.

\section{Waist and neck circumferences}

The circumferences measurement will be performed with the patients in an anatomical position, using a non-elastic tape measure, parallel to the ground, with an accuracy of $0.1 \mathrm{~cm}$. The measuring points will be standardized as following, according to scientific literature. For the waist's circumference, the measuring in the middle between the inferior border of the last rib and the iliac crest will be used; fixating the zero point tape to the thorax from the axillary line's level, which is going to be used (axillary, xiphoidian or abdominal). The neck's circumference will be taken horizontally on the cricoid cartillage. ${ }^{(40-42)}$

\section{Plethysmography}

Pulmonary function tests will be performed at the Pulmonary Function Laboratory of the Cascavel Lung Institute through the Jaeger Vmax plethysmograph (Care Fusion - Pneumotach ID 0-25A6-221C-1A9A, Germany) following the national guidelines for conducting pulmonary function of SBPT ${ }^{43}$ and $\mathrm{ERS}^{(44-46)}$. All patients will be plethysmography in the sitting position according to standards established by the ATS/ERS. The diagnosis of COPD was confirmed as a post-bronchodilator forced expiratory volume in 1s (FEV1)/with a forced vital capacity ratio of 0.7 . The severity of the disease was graded according to the Global Initiative for Chronic Obstructive Lung Disease guideline. ${ }^{(39)}$

The data collected from plethysmograph will be forced vital capacity (FVC), forced expiratory volume in 1s (FEV1), total lung capacity (TLC), FEV1/FVC ratio, inspiratory capacity (IC), residual volume (RV) and resistance of upper airway (RAW).
COPD was defined as an FEV1/FVC ratio $<75 \%$; the severity was determined based on the percent of predicted FEV1.

\section{BODE index}

The BODE index integrates four factors relevant to the respiratory, perceptive, and systemic aspects of COPD, including $\mathrm{BMI}$, the degree of airflow obstruction, functional dyspnea, and exercise capacity; the latter is assessed with a 6-min walking test (6MWD). Each component is graded and a composite score from 1 to 10 is obtained, with higher scores indicating greater severity. The index score reflects the impact of both pulmonary and extrapulmonary factors on the prognosis and survival in COPD. ${ }^{(47)}$

\section{Sleep study parameters}

An attended nocturnal polysomnography will be performed using standard techniques in all patients at their approximate routine sleeping hours. Via the Alice 5 system, (Philips Respironics, Pittsburgh, PA, USA), the following channels will be registered: electroencephalogram (C3/A2, C4/A1, 01/A2, 02/A1), bilateral electrooculogram (EOG), submental and anterior tibialis electromyogram (EMG), EKG, rib cage and abdominal motion by inductive plethysmography, body position, nasal cannula/pressure transducer system for respiratory monitoring and oximetry with digital signal extraction (Masimo ${ }^{\circledR}$, Irvine, CA, USA).

The PSG exams will be manually analyzed according to the criteria of Rechtschaffen and Kales by an expert polysomnographic technologist. ${ }^{48}$ Arousals and respiratory events were scored using the American Academy of Sleep Medicine criteria. ${ }^{49}$ Respiratory events consisted of apneas, defined as a reduction in air flow $=90 \%$ associated with sustained chest and abdominal effort, hypopneas, defined as a reduction in air flow $=50 \%$ associated with sustained chest and abdominal effort, and RERA events, defined as inspiratory flow limitation events followed by an EEG arousal which was not associated with any other sleep disturbance. The apnea-hypopnea index (AHI) included obstructive apneas and hypopneas linked to oxyhemoglobin drop $=4 \%$. The respiratory disturbance index (RDI) consisted of the addition of the RERA hourly index to the AHI. ${ }^{(50,51)}$

Sleep quality will be objectively assessed in all study patients by obtaining the following measurements: sleep efficiency (time asleep/time in bed), sleep architecture, latency to persistent sleep and the arousal index.

\section{Pittsburgh Sleep Questionnaire Index}

The Pittsburgh Sleep Questionnaire Index (PSQI) will be used to assess the quality of sleep. The PSQI consists of 19 items and provides a well-validated global index of sleep quality over the previous 1-month time interval. PSQI greater than 5 is generally considered an indicator of poor sleep quality. ${ }^{(52)}$ 


\section{Epworth Sleepiness Scale}

The Epworth Sleep Scale (ESS) is a self-applicable questionnaire, based in questions about situations of excessive sleepiness involving daily activities. The subjects will be oriented to classify in a scale of 0 to 3 the probability of feeling sleepy or falling asleep in eight different situations. Being zero no probability of falling asleep at all, one small probability of it happening, two moderate probability and three high probability. ${ }^{(53,54)}$

\section{Berlin Questionnaire}

The Berlin Questionnaire (BQ) is a clinical questionnaire of acknowledged efficacy on verifying risk for OSA. This instrument is composed by ten items, organized in three categories relative to snore and OSA (containing 5 items), daytime sleepiness (4 items) SH and obesity ( 1 item). Any bolded answer is considered positive. The punctuation is divided in three categories in which category 1 is considered positive with two or more positive answers for questions 1 to 5 . Category 2 is considered positive with two or more positive answers to questions 6 to 8 and category 3 is considered positive if the answer for question 9 is yes or the BMI is over $30 \mathrm{~kg} / \mathrm{m}^{2}$. Two or more positive categories indicate a high risk for OSA. ${ }^{(55)}$

\section{STATISTICAL ANALYSIS}

Data of patients will be presented as mean \pm standard deviation, in the appropriate text and tables. Fisher's exact test was used for categorical variables and independent sample t-test was used for continuous variables to compare patients with overlap syndrome against those without it. Correlation analysis and multivariate linear regression analysis were used to determine the relationship between the demographic, anthropometric, polygraphic and laboratory parameters with AHI. The correlations were scored as weak ( $r=0-0.49)$, moderate $(r=0.5-0.74)$ or strong $(r=0.75-1)$. The data was assessed in $95 \%$ confidence interval and the statistical significance value was $P<0.05$. All analyses were performed using the Statistical Package for the Social Sciences version 19.0 (SPSS Inc, Chicago, IL, USA). A p-value $<0.05$ was considered statistically significant.

\section{Sample size}

The study by Soler et al. (2016) ${ }^{7}$ identified a $65.9 \%$ prevalence of Overlap syndrome in patients with COPD, according to $\mathrm{AHI}$ greater than 5 events per hour (the effect of expected size). Using the standard deviation observed by the study of 20.8 events / $h$ and assuming $\alpha=0.05$ and power $=80 \%$, the required sample was estimated in 33 patients. Six patients $(20 \%)$ will be added to compensate for possible sample losses.

\section{FINAL CONSIDERATIONS}

Overlap syndrome, or the coexistence of OSA and COPD, are common inflammatory systemic disorders with increasing prevalence that causes more significant oxygen disturbances than does either of these disorders alone. ${ }^{(20,56)}$ Patients have significant reductions in sleep efficiency, oxygen desaturations, as well as increased arousal index and daytime sleepiness. ${ }^{(20)}$

When they coexist, their syndrome leads to increased disease severity, morbidity, and mortality. Treating OSA in patients with COPD can improve disease outcomes, so clinicians must strive to recognize patients who are prone to sleep-disordered breathing, especially those with airway disease, so they may be treated appropriately.

The quality of sleep in COPD is compromised by several factors, and additional studies on this topic are needed to fully assess the relationship between COPD and OSA, towards early diagnosis and to identify therapeutic interventions that may improve overall quality of life and survival in patients with COPD.

\section{Description of risks}

There will be no risks for included patients.

\section{AUTHOR'S CONTRIBUTION}

JCMO and LVFO defined the concept of the study, created the hypothesis, and wrote the original proposal. EFO, ASS, JJU, EAP and LVFO contributed significantly to writing this proposal. JJU, EAP, IRS, ASS, and LVFO were involved in the critical review of the manuscript. JCMO, RAOP and LVFO wrote this protocol role, with the contribution of all co-authors. All authors read and approved the final manuscript.

\section{CONFLICTS OF INTEREST}

The author(s) declare that they have no competing interests.

\section{AUTHOR DETAILS}

${ }^{2}$ Lung Institute, Cascavel, (PR), Brasil. ${ }^{3}$ Faculdade de Ciências Médicas da Santa Casa de São Paulo, São Paulo, (SP), Brasil.

\section{REFERENCES}

1. National Institutes of Health (NIH), National Heart Lung and Blood Institute (NHLBI), World Health organization (WHO). Global Strategy for the Diagnosis, Management, and Prevention of Chronic Obstructive Pulmonary Disease (GOLD). NHLBI/WHO Workshop Report. www. goldcopd.com/workshop/ index.html. 2007 and update in 2008.

2. Fabri LM, Hurd SS, For the GOLD Scientific Committee. Editorial: Global strategy for the diagnosis, management and prevention of COPD: 2003 update. Eur J Respir 2003; 22:1-2.

3. Jardim JRB, Oliveira JCA, Nascimento O. II Consenso Brasileiro sobre Doença Pulmonar Obstrutiva Crônica. J Bras Pneumol 2004; 30: S1-S42.

4. Lozano R, Naghavi M, Foreman K, et al. Global and regional mortality from 235 causes of death for 20 age groups in 1990 and 2010: a systematic analysis for the Global Burden of Disease Study 2010. Lancet 2012; 380(9859): 2095-128.

5. Mathers CD, Loncar D. Projections of global mortality and burden of disease from 2002 to 2030. PLoS Med 2006; 3(11): e442.

6. Flenley DC. Sleep in chronic obstructive lung disease. Clin Chest Med, 6 (1985), pp. 51-610 
7. Soler X, Gaio E, Powell FL, Ramsdell JW, Loredo JS, Malhotra A et al. High prevalence of obstructive sleep apnea in patients with moderate to severe chronic obstructive pulmonary disease. Ann Am Thorac Soc. 2015;12(8):1219-25.

8. Soler-Cataluna JJ et al. Severe acute exacerbations and mortality in patients with chronic obstructive pulmonary disease. Thorax 2005;60(11):925-931.

9. Jemal A, Ward E, Hao Y, Thun M. Trends in the leading causes of death in the United States, 1970- 2002. JAMA 2005; 294(10): 1255-9.

10. Mannino DM, Gagnon RC, Petty TL, Lydick E. Obstructive lung disease and low lung function in adults in the United States: data from the National Health and Nutrition Examination Survey, 1988-1994. Arch Intern Med 2000; 160(11): 1683-9.

11. Young T, Palta M, Dempsey J, Skatrud J, Weber S, Badr S. The occurrence of sleep-disordered breathing among middle-aged adults. N Engl J Med 1993; 328(17): 1230-5.

12. Chaouat A, Weitzenblum E, Krieger J, Ifoundza T, Oswald M, Kessler R. Association of chronic obstructive pulmonary disease and sleep apnea syndrome. Am J Respir Crit Care Med 1995; 151(1): 82-6.

13. Shepard JW, Jr., Garrison MW, Grither DA, Evans R, Schweitzer PK. Relationship of ventricular ectopy to nocturnal oxygen desaturation in patients with chronic obstructive pulmonary disease. Am J Med 1985; 78(1): 28-34.

14. Bradley TD, Rutherford R, Grossman RF, et al. Role of daytime hypoxemia in the pathogenesis of right heart failure in the obstructive sleep apnea syndrome. Am Rev Respir Dis 1985; 131(6): 835-9.

15. Weitzenblum E, Krieger J, Apprill M, et al. Daytime pulmonary hypertension in patients with obstructive sleep apnea syndrome. Am Rev Respir Dis 1988; 138(2): 345-9.

16. Trask $\mathrm{CH}, \mathrm{Cree} \mathrm{EM}$. Oximeter studies on patients with chronic obstructive emphysema, awake and during sleep. N Engl J Med 1962;266:639-642.

17. Pierce AK et al. Respiratory function during sleep in patients with chronic obstructive lung disease. J Clin Invest 1966; 45 (5):631-636.

18. Hawrylkiewicz I et al. Pulmonary haemodynamics in patients with OSAS or an overlap syndrome. Monaldi Arch Chest Di. 2004;61(3):148-152

19. Owens RL and Malhotra A. Sleep-disordered breathing and COPD: The overlap syndrome. Resp Care 2010;55(10): 1333-1346

20. Sanders $\mathrm{MH}$ et al. Sleep and sleep-disordered breathing in adults with predominantly mild obstructive airway disease. Am J Respir Crit Care Med 2003;167(1):7-14

21. Marin JM et al. Outcomes in patients with chronic obstructive pulmonary disease and obstructive sleep apnea: the overlap syndrome Am J Respir Crit Care Med 2010 Aug 1;182(3):325-31. doi: 10.1164/rccm.20091218690C. Epub 2010 Apr 8.

22. Lavie $P$ et al. Mortality in sleep apnea patients: a multivariate analysis of risk factors. Sleep 1995;18(3):149-157

23. McNicholas WT and Fitzgerald MX. Nocturnal deaths among patients with chronic bronchitis and emphysema. BMJ (Clin Res Ed) 1984; 289(6449):878

24. Mermigkis $C$ et al. Health-related quality of life in patients with obstructive sleep apnoea and chronic obstructive pulmonary disease (overlap syndrome). Int J Clin Pract 2007;61(2): 207-211

25. Celli BR, MacNee W; ATS/ERS Task Force. Standards for the diagnosis and treatment of patients with COPD: a summary of the ATS/ERS position paper. Eur Respir J 2004;23:932-46.

26. Mannino DM, Buist AS. Global burden of COPD: risk factors, prevalence, and future trends. Lancet 2007;370:765-73.

27. Peppard PE, Young $\mathrm{T}$, Barnet $\mathrm{JH}$, et al. Increased prevalence of sleepdisordered breathing in adults. Am J Epidemiol 2013;177:1006-14.

28. Celli BR, Halbert RJ, Isonaka S, et al. Population impact of different definitions of airway obstruction. Eur Respir J 2003;22:268-73.
29. Lévy P, Kohler M, McNicholas WT, et al. Obstructive sleep apnoea syndrome. Nature Reviews Disease Primers 2015;1:1-20.

30. Tufik S, Santos-Silva R, Taddei JA, et al. Obstructive sleep apnea syndrome in the Sao Paulo Epidemiologic Sleep Study. Sleep Med 2010;11:441-6.

31. Calderón E, Carmona C, Arenas M, Fuentes MA, Sánchez A, Capote F. Estudio comparativo de pacientes con enfermedad pulmonar obstructiva crônica con y sin síndrome de apneas obstructivas durante el sueno. Arch Bronconeumol. 1999;35: 539-43.

32. Gullu Z, Itil O, Oztura I, Aslan O, Ceylan E, Baklan B et al. Kronik obstruktif akciger hastaligi ve obstruktif uyku apne sendromu birlikteligi (overlap sendromu). Turk Toraks Dergisi. 2002;2: 161-7.

33. Venkateswaran S, Tee A. Overlap syndrome between chronic obstructive pulmonary disease and obstructive sleep apnoea in a Southeast Asian teaching hospital. Singapore Med J. 2014 Sep;55: 488-92.

34. Kokturk O, Tathcıoglu T, Firat H, Cetin N. "Overlap Sendromu” kronik obstruktif akciger hastalarında obstruktif sleep apne sendromu. Tuberkuloz Ve Toraks. 1996;44: 187-92.

35. Resta O, Foschino Barbaro MP, Brindicci C, et al. Hypercapnia in overlap syndrome: possible determinant factors. Sleep Breath. 2002;6: 11-8.

36. McNicholas WT. Chronic obstructive pulmonary disease and obstructive sleep apnea: overlaps in pathophysiology, systemic inflammation, and cardiovascular disease. Am J Respir Crit Care Med 2009;180:692-700.

37. Vandenbroucke JP, von Elm E, Altman DG, Gøtzsche PC, Mulrow CD, Pocock SJ, et al. STROBE Initiative. Strengthening the Reporting of Observational Studies in Epidemiology (STROBE): explanation and elaboration. Epidemiology. 2007; 18(6):805-35

38. Rabe KF, Hurd S, Anzueto A, Barnes PJ, Buist SA, Calverley P, et al; Global Initiative for Chronic Obstructive Lung Disease. Global strategy for the diagnosis, management, and prevention of chronic obstructive pulmonary disease: GOLD executive summary. Am J Respir Crit Care Med 2007; 176:532-55

39. Global Initiative for Chronic Obstructive Lung Disease (GOLD). Global strategy for the diagnosis, management and prevention of COPD. Available at: http://www.goldcopd. org/ (accessed 08 Dec 2016).

40. Hingorjo MR, Qureshi MA, Mehdi A. Neck circumference as a useful marker of obesity: A comparison with body mass index and waist circumference. J Pak Med Assoc 2012;62(1):36-40.

41. Vallianou NG, Evangelopoulos AA, Bountziouka V, et al. Neck circumference is correlated with triglycerides and inversely related with $\mathrm{HDL}$ cholesterol beyond BMI and waist circumference. Diabetes Metab Res Rev 2013;29:90-97.

42. Gabrielsen AM, Lund MB, Kongerud J, Viken KE, Røislien J, Hjelmesæth J. The relationship between anthropometric measures, blood gases, and lung function in morbidly obese white subjects. Obes Surg. 2011 Apr;21(4):485-91.

43. Diretrizes para Testes de Função Pulmonar. J Pneumol 2002; 28: S166206.

44. American Thoracic Society. ATS statement: guidelines for the six-minute walk test. Am J Respir Crit Care Med 2002; 166:111-17.

45. Quanjer PH, Tammeling GJ, Cotes JE, Pedersen OF, Peslin R, Yernault JC: Lung volumes and forced ventilatory flows. Report Working Party Standardization of Lung Function Tests, European Community for Steel and Coal. Official Statement of the European Respiratory Society. Eur Respir J Suppl 1994, 7(6):1197-8.

46. Pereira CAC: II Consenso Brasileiro de Espirometria. J Pneumol 2002, 28(3):S1-S82.

47. Celli BR, Cote CG, Marin JM, et. al. The body-mass index, airflow obstruction, dyspnea and exercise capacity index in chronic obstructive pulmonary disease. N Engl J Med. 2004 Mar 4;350(10):1005-12. 
48. Rechtschaffen A, Kales A: A manual of standardized terminology: techniques and scoring system for sleep stages of human subjects. Los Angeles: Brain Information Service/Brain Research Institute; 1968. Psychiatry and Clinical Neurosciences. 2001; 55:305-310.

49. Iber C, Ancoli-Israel S, Chesson Jr A, Quan S: The AASM manual for the scoring of sleep and associated events: rules, terminology and technical specifications. Westchester: merican Academy of Sleep Medicine; 2007.

50. American Academy of Sleep Medicine. The international classification of sleep disorders: diagnostic and coding manual. 2nd ed. Westchester (IL): Author; 2014

51. International Classification of Sleep Disorders, 3rd ed. Darien, IL, American Academy of Sleep Medicine, 2014.
52. Buysse DJ, Reynolds CF III, Monk TH, Berman SR, Kupfer DJ. The Pittsburgh Sleep Quality Index: a new instrument for psychiatric practice and research. Psychiatry Res 1989;28:193-213.

53. Murray WJ: A new method for measuring daytime sleepiness: the Epworth sleepiness scale. Sleep. 1991;14(6):540-545.

54. Murray WJ: Reliability and factor analysis of Epworth sleepiness scale. Sleep. 1992; 15(4):376-81.

55. Netzer NC, Stoohs RA, Netzer CM, Clark K, Strohl KP: Using the Berlin Questionnaire to identify patients at risk for the sleep apnea syndrome. Ann Intern Med. 1999, 131(7):485-491.

56. Becker HF, Piper AJ, Flynn WE, et al. Breathing during sleep in patients with nocturnal desaturation. Am J Respir Crit Care Med. 1999;159(1):112-118. 19. 